OPEN ACCESS

Edited by:

Bin Zhou,

Hunan University, China

Reviewed by:

Yixun Xue,

Taiyuan University of Technology,

China

Yan Zhang,

Guangdong University of Finance and

Economics, China

*Correspondence:

Xueqian $\mathrm{Fu}$

fuxueqian@cau.edu.cn

Specialty section: This article was submitted to Process and Energy Systems Engineering,

a section of the journal Frontiers in Energy Research

Received: 19 December 2021 Accepted: 27 December 2021

Published: 03 February 2022

Citation:

Fu X, Yang K, Li G and Zeng D (2022) Research on the Trading Arrangement and Clearing Model of Medium- and Long-Term Inter-Provincial Markets Considering Security Constraints. Front. Energy Res. 9:839108. doi: 10.3389/fenrg.2021.839108

\section{Research on the Trading Arrangement and Clearing Model of Medium- and Long-Term Inter-Provincial Markets Considering Security Constraints}

\author{
Xueqian $\mathrm{Fu}^{1 *}$, Kaitao Yang ${ }^{1}$, Guodong $\mathrm{Li}^{2}$ and Dan Zeng ${ }^{3}$ \\ ${ }^{1}$ College of Information and Electrical Engineering, China Agricultural University, Beijing, China, ${ }^{2}$ Beijing Power Exchange Center, \\ Beijing, China, ${ }^{3}$ China Electric Power Research Institute, Nanjing, China
}

In the process of deepening market-oriented reform, the most important thing in China is building a national unified electricity market system under a high renewable energy penetration scenario. The new round of market-oriented reform proposes to establish an inter-provincial electricity-trading arrangement. Due to imperfect market mechanisms and immature inter-provincial electricity markets, we investigate the electricity market operation system of the national unified electricity market. Existing transmission commitments, trading cycles, market participants, strategic bids, and clearing and settlement mechanisms are analyzed for inter-provincial medium- and long-term markets. For security reasons of interchange schedules, we propose a novel interprovincial market-clearing model based on static security regions, which consider inter-provincial electricity transmissions and direct current power flows. The proposed market-clearing model is simulated and verified in a 3-bus system and an IEEE 30-bus system. The proposed clearing model and rational market theory are conducive not only to promoting renewable energy consumption but also to improving the linkages between inter-provincial medium- and long-term markets.

Keywords: unified power market, inter-provincial transaction, trading arrangement, market clearing, bid price

\section{INTRODUCTION}

Electricity market reform is steadily advancing in China. In 2021, the Central Committee for Comprehensively Deepening Reforms reviewed and approved the rules for a national unified electricity market system (NUEMS). All these indicate that China will accelerate the construction of inter-provincial electricity markets and launch new electricity-trading arrangements for medium- and long-term (M-L) markets to optimize renewable energy resources. In addition, attention has also been paid to the multi-energy market for different types of energy (Liu et al., 2019).

Eastern China has high energy consumption, while Western China has large energy production. More than $80 \%$ of the overall capacity is installed in the Midwest and Northern regions, and more than $70 \%$ of nationwide consumed energy is concentrated in the southeast coastal and central regions (Xia et al., 2020). At the present time, China establishes power systems with high-permeability renewable sources, which include photovoltaic, wind generator, and hydrogen production (Zhang et al., 2021), and the scale and scope of electricity markets are growing. In this case, we should induct optimal allocation of inter-provincial renewable energy. It is extremely important and urgent to 
increase the amount of energy consumption from renewables and solve the contradiction problem caused by the gradual expansion of power supply and demand (Guo et al., 2019). Therefore, it is almost an inevitable requirement to build a NUEMS to reduce the carbon footprint at the provincial level.

An initial trading manner has been established for interprovincial M-L markets in China, and many scholars have also studied the key issues in the construction of a NUEMS from different aspects. Guo et al. described the background, motivation, and history of China power market reforms and made several recommendations for the forward market, spot market, and ancillary service market (Guo et al., 2020). Jiang et al. pointed out that China needs to establish a power balance mechanism suitable for M-L power-trading management. They presented a two-tier bidding model for power producers to obtain a reasonable bid price to an independent market operator (Jiang et al., 2019). Zeng et al. simplified the inter-provincial transmission model through a clustering algorithm and presented an inter-provincial electricity-trading arrangement, which played major roles in ensuring that both independent power producers and provincial power marketers participate in inter-provincial trading (Zeng et al., 2020a). Zeng et al. established a market-clearing mechanism combining intraand inter-provincial electricity markets and made a three-stage path plan for hierarchical, loosely coupled, and tightly coupled clearing models (Zeng et al., 2020b). Song et al. analyzed the coordination mechanism between the futures market and the spot market based on the current status of a provincial spot market in China (Song et al., 2020).

The low utilization rate of renewable energy is a key issue hindering electricity market reform. Zeng et al. took Yunnan as an example and analyzed the impacts of bilateral transaction on the collaboration between renewable electricity generation and thermal power generation (Zeng et al., 2015). Liu et al. took China's Guangdong Province as an example to study the economic evaluation of renewable energy under the conditions of power marketization. They believed that both coal price and power demand have impacts on green power marketing activities (Liu et al., 2020). Abhyankar et al. took China's Southern power system as an example and examined the impact of large-scale market operations on the total cost curve, production costs, and carbon dioxide emissions. They believed that the electricity market can reduce the carbon dioxide emissions of the Southern Power Grid by 10\% (Abhyankar et al., 2020). Cai et al. constructed a renewable energy trading arrangement for monthly inter-provincial trading, and consumer demand and renewable energy were matched by introducing "inter-provincial traders" (Cai et al., 2021).

The inter-provincial electricity transmission capacity is crucial to the consumption of renewable energy. Li et al. conducted a research on "source-network-load" interaction to increase the amount of energy consumption from renewables. They established a renewable energy adjustment calculation model to analyze the potential of the "Three-North" renewable energy project in China (Li et al., 2019). The energy transition of the power industry has a significant role in promoting renewable energy development. Liu et al. studied the theoretical basis of market design via institution-economics-technology-behavior. They made suggestions on market design from both market and policy aspects (Liu et al., 2021). The open-access same-time information system is a necessary condition for the process of establishing a competitive power market. Jin et al. adopted a game model to analyze the behavior of market participants and calculated different market-clearing prices (MCPs) under different information disclosure scenarios (Jin et al., 2021). The security region method is popular in power system security analysis (Xiao et al., 2019). The security region method can judge power system security involving a variety of security constraints (Jiang et al., 2020). The static security region describes the operating area that satisfies the system power flow equation, unit active power security constraint, branch power transmission constraint, and so on (Jiang et al., 2019). The security region method was adequately studied in (Yu et al., 2020). The shortcomings of existing works are as follows: 1) The existing works in Li et al. (2019), Liu et al. (2021), Jin et al. (2021) focus on the billing and clearing models of inter-provincial trade in China, but the security region has not been given enough credit by the market operator. 2) The current market mechanism considers no security constraint in the early stage of market clearing, and many trading schedules are unreached due to grid security issues. It is conducive to develop a security-constrained trading schedule for the construction of inter-provincial electricity markets.

Two innovations of this paper are listed. 1) We propose a novel inter-provincial electricity-trading arrangement for M-L markets considering market linkages at different timescales. The proposed electricity-trading arrangement can support daily trading divided from monthly curves, and the division realizes the linkages between spot and M-L markets. This mechanism can promote interaction to increase the amount of energy consumption from wind farms in provincial electricity markets. 2) To the best of our knowledge, static security constraints are considered in clearing of an inter-provincial electricity-trading system for the first time. A market-clearing model based on security region can ensure that clearing results pass the security check of a power-dispatching center.

The rest of this paper is organized as follows: We review the current construction situation of the NUEMS in China Power Market Outlook. Inter-Provincial Electricity-Trading Arrangement introduces an inter-provincial M-L market electricity-trading arrangement. Market-Clearing Model describes the market-clearing model, and Simulation Analysis gives an example analysis. Finally, Discussion gives a summary.

\section{CHINA POWER MARKET OUTLOOK}

China has formed a unified electric grid consisting of seven regional power grids through ultra-high-voltage alternating current-direct current (DC) transmission lines. This has laid a physical foundation for the NUEMS. Two NUEMS have been established in Beijing and Guangdong, and it marks that two-tier power market trading platforms have been developed in China. 
TABLE 1 | Functional positioning of a two-tier electricity market.

\section{Functional positioning}

Market positioning

Market player

Market operation agency
Inter-provincial transaction

Resource allocation market

Power generation company

Electricity sales company

Large power customer

Power grid company

NEPTC

NEPDCC

Power regulatory agency
Intra-provincial transaction

Power balance market

Power generation company

Electricity sales company

Power customer

Power grid company, etc.

PEPTC

PEPDC

Power regulatory agency
The functional positioning (Zhang and Shi, 2020) of China's twotier power market is listed in Table $\mathbf{1}$.

With respect to the inter-provincial market in China, contract trading is a classic and reliable method for M-L markets. Interprovincial transaction includes incremental spot transactions of renewable energy. The inter-provincial contract transaction model consists of three stages. In the first stage, market participants negotiate the energy, time, and price to form a preliminary trading schedule. In the second stage, the negotiated schedule is submitted to the system operator for safety check. The third stage is to sign the contract and execution the schedule. The problem of surplus renewable energy and electricity shortage are solved in the spot market.

Before we start spot trading, the intra-provincial market is dominated by the trading arrangement of M-L markets. In 2019, China established eight provincial spot markets in provinces such as Guangdong and Shandong. The intra-provincial power market in China has gradually transitioned to a provincial electricity spot market (Ding et al., 2020). As matters stand, the development process of electricity market is different in different provinces, and the trading manner is diverse in different provinces. Each province has formed its own contract management system and electricity market operation system, and there exist interprovincial trade barriers. Among the provincial spot markets, the West Inner Mongolia Power Grid adopts a centralized market model, while other provinces adopt a decentralized market model. When designing electricity-trading arrangements, each province has poorly considered inter-provincial market connections in terms of functional positioning, trading cycle, and price mechanism.

The power market is different from ordinary commodity markets. It has the dual attributes of "grid operation" and

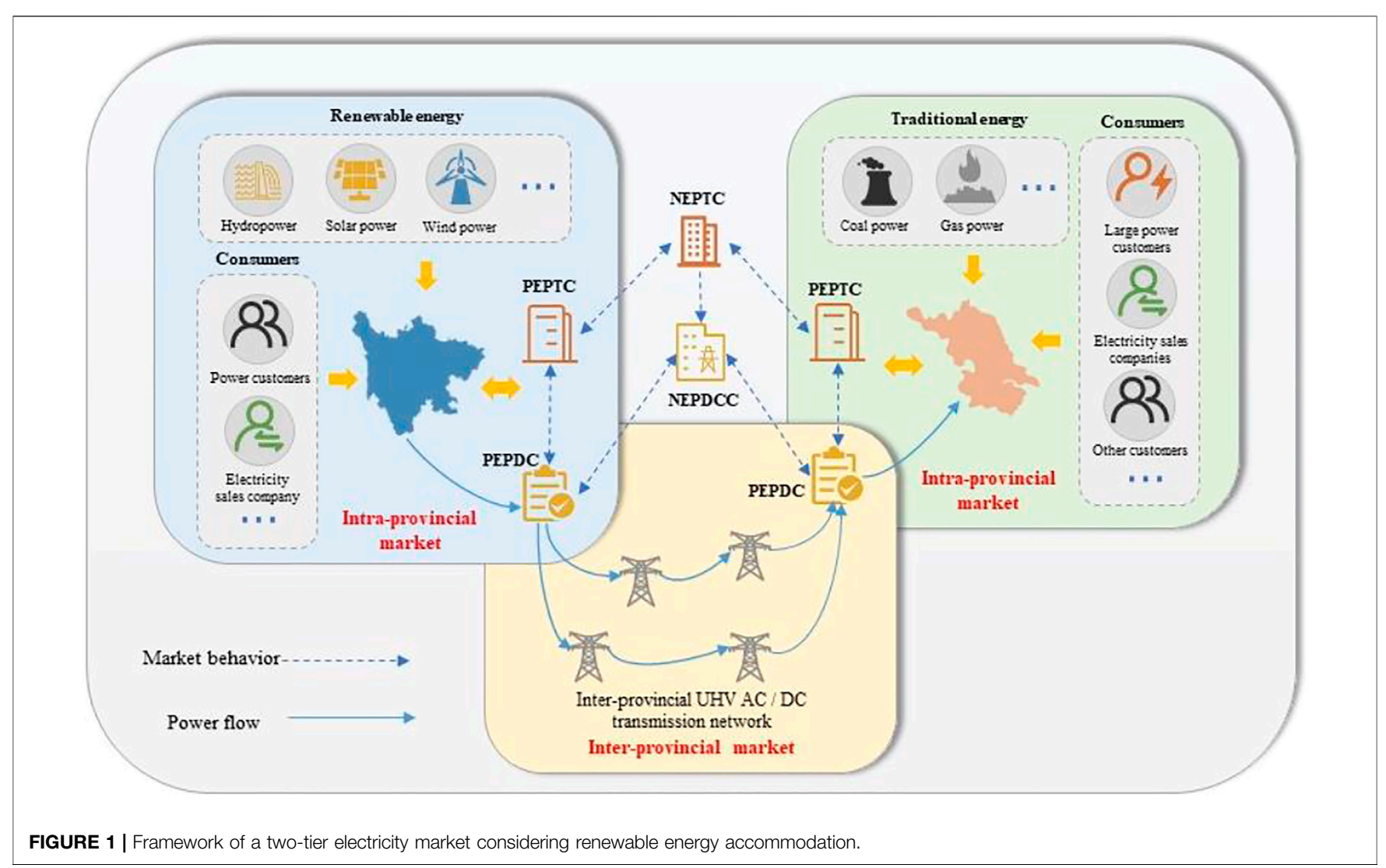


TABLE 2 | Types of electrical bid template.

\begin{tabular}{|c|c|c|}
\hline Template & Characteristic & Application scenarios \\
\hline $\begin{array}{l}\text { Ordinary hourly } \\
\text { bids }\end{array}$ & The generator/consumer side bids the price, power, and time period at the same time & Various electricity power units and power customers \\
\hline Block bids & $\begin{array}{l}\text { Quotation of a variety of quantity-price combinations. Following the principle of "all deals or } \\
\text { no deals" }\end{array}$ & Nuclear power units or large thermal power units \\
\hline Flexible hourly bids & Only the power generation side bids the segment electricity and the corresponding price & $\begin{array}{l}\text { Flexible power units such as hydraulic and thermal power } \\
\text { units }\end{array}$ \\
\hline
\end{tabular}

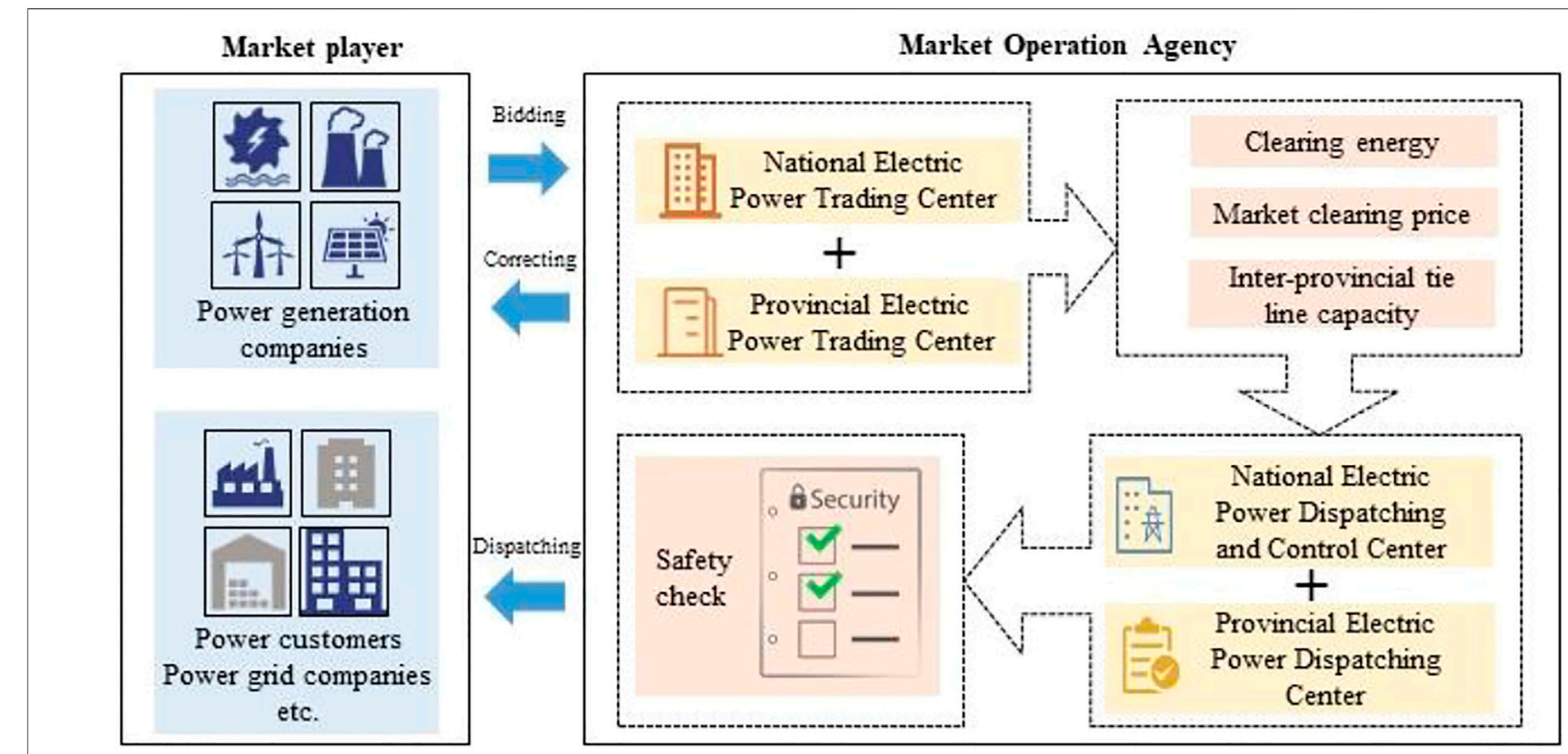

FIGURE 2 | Clearing mechanism of a two-tier electricity market.

"market transaction." Although an electricity-trading arrangement has been basically established in inter-provincial markets, there are still some problems. The current M-L transactions between provinces adopt an unconstrained clearing method, which causes some trading schedules failing to complete trading. Although some provinces have started spot market construction, the current China M-L and spot markets are still separated. The M-L markets are still dominated by annual and monthly trading strategies. There is still some distance between the mature and open electricity markets. The manner of linking the power system and the power market determines the market architecture. When planning and constructing the unified national power market, China must consider both grid operation and market architecture and design the market mechanism reasonably.

\section{INTER-PROVINCIAL ELECTRICITY-TRADING ARRANGEMENT}

At present, China's inter-provincial electricity-trading arrangement is not perfect, and there are still some deficiencies in the market-pricing mechanism, development mechanism, and other aspects. In the context of the new round of electricity reform, we propose an inter-provincial electricity-trading arrangement that can promote the consumption of clean energy. The following section will introduce the market framework, market participants, trading cycles, bidding strategies, clearing mechanisms, and settlement mechanisms, respectively.

\section{Market Framework}

The market participants include market operators and market players in inter-provincial M-L markets. Market operators include the power-trading center and power-dispatching center and the power regulatory agency. Market players include various power generation companies, electricity sales companies, large power customers, and power grid companies.

The National Electric Power Trading Center (NEPTC) is responsible for electricity-trading management in $\mathrm{M}$-L interprovincial markets, and the Provincial Electric Power Trading Center (PEPTC) is responsible for electricity-trading management in M-L intra-provincial markets. In the initial stage of market construction, the PEPTC can act as agents of 


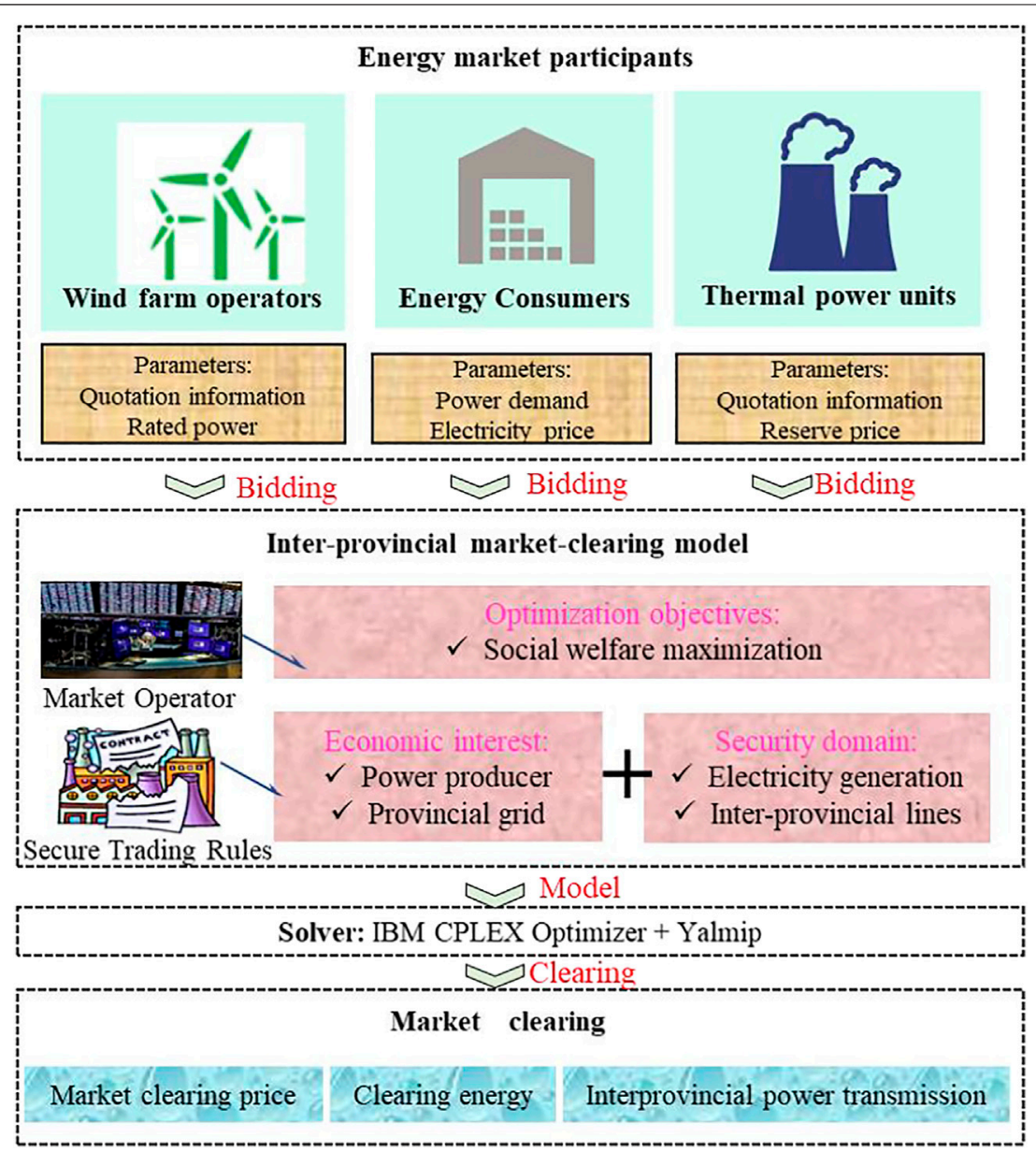

FIGURE 3 | Solution process of an inter-provincial market-clearing model.

provincial market players to participate in inter-provincial markets. The power generation companies in power-sending provinces include wind power, photovoltaic, hydropower, and other power producers. Some inter-provincial power producers and large power customers can directly participate in inter-provincial market transaction through the NEPTC. Figure 1 shows the framework of a two-tier electricity market considering renewable energy accommodation.

It can be concluded that the power trading of the M-L interprovincial market is mainly composed of the following three steps: intra-provincial market pre-clearing and inter- and intraprovincial market clearing. Here we focus on the inter-provincial electricity-trading arrangement. The M-L inter-provincial market mechanism should meet the annual national priority power generation schedules. This part of electricity is dominated by renewable energy generation, and the electricity price is determined using market-oriented methods. Monthly incremental transactions are carried out on the basis of the annual national priority generation schedules. First, the PEPTC determines the monthly sufficient or surplus electricity generation capacity of the province and breaks a monthly electricity curve down by day. Second, the NEPTC organizes the inter-provincial M-L market clearing in consideration of security constraints and publishes the clearing energy and MCP. Finally, the National Electric Power Dispatching and Control Center (NEPDCC) and Provincial Electric Power Dispatching Center (PEPDC) conduct a safety check on the constrained trading schedule.

\section{Trading Cycle}

The inter-provincial market trading cycles can be divided into annual trading, monthly trading, and daily trading. In addition, the linkages between $\mathrm{M}-\mathrm{L}$ and spot markets are realized via converting monthly trading and daily trading. The trading schedule of $\mathrm{M}-\mathrm{L}$ and spot markets includes the following steps. 1) Inter-provincial annual trading is arranged to ensure renewable energy trading priority. 2) Interprovincial monthly trading is arranged based on the annual trading schedule. 3) In the inter-provincial monthly market, the monthly transacted electricity quantity is $70 \%$ of the total power demand for the next month, and the remaining $30 \%$ can be traded in the spot market. Each province can determine the proportion of the transacted electricity quantity in 


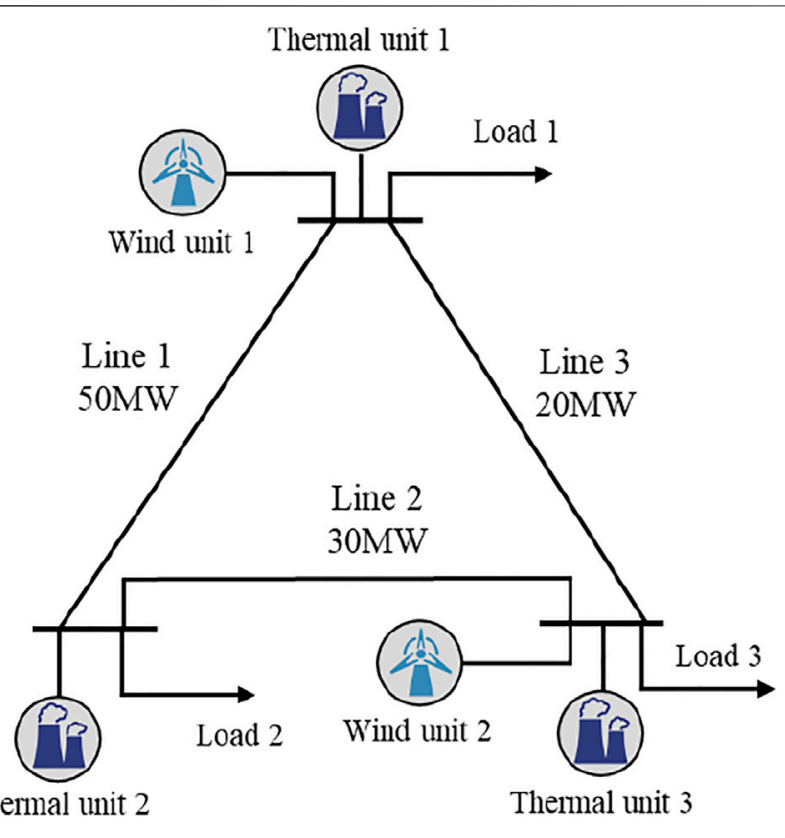

FIGURE 4 | Structure diagram of the 3-bus power system under study.

different markets according to its own actual situation. 4) Market-based balance management is carried out in spot markets, thereby realizing the linkages between the M-L and spot markets.

\section{Transaction Subject}

The spot market transaction is subject to different timescales. In order to link the M-L and spot markets, the timescale of the M-L market must be consistent with that of the spot market. Therefore, the subject matter of the monthly trading should be the electricity quantity decomposed by the curve of the next month. According to the formation method of the time-sharing power curve, there are two categories of transaction curves, namely, a standardized transaction curve and a custom transaction curve. A standardized trading curve includes the annual, monthly, and weekly decomposition curves, which are formulated and released by the market operators according to grid operating conditions. In terms of custom trading curve, market players decompose contract electricity into time-sharing power according to their own power generation and load characteristics. This curve decomposition method does not limit the proportion of electricity quantity in each period.

In the initial stage of a NUEMS, the market mechanism is immature. The physical execution of the M-L transaction decomposition curve may cause system congestion and peak shaving resource shortage. These are not conducive to the consumption of renewable energy. Therefore, the decomposed curve should be executed in a financial way, and it can be executed in a physically way when the market is mature enough.

\section{Bid Mechanism}

The bid mechanism of the China electricity market is not perfect, and the bid mechanisms are different in different provinces. Therefore, China can study the bid mechanisms of other countries. Different types of electrical bid template study are listed in Table 2.

In the initial stage of the inter-provincial $\mathrm{M}-\mathrm{L}$ market construction, each PEPTC can offer a bilateral bidding model to market participants in inter-provincial electricity transactions. Provincial market participants still send the bid price and energy to the PEPTC in accordance with the intra-province bid mechanism. Inter-provincial market participants send the bid price and energy to the NEPTC through the hourly rate bidding schedule. The provincial electricity providers provide generation offers, ancillary service charges, and bidding price. The provincial electricity consumers provide load bids.

\section{Clearing Mechanism}

The inter-provincial M-L transactions are carried out through centralized trading. Market participants must report to the PEPTC on time. The report should contain the monthly electricity and electricity price with a curve. The PEPTC summarizes the information throughout the entire province and sends it to the NEPTC. Then, the NEPTC clears the transaction. An inter-provincial market-clearing model can involve multiple constraints, which include the operation of provincial power grids and inter-provincial tie-line transmission capacity. Finally, the NEPDCC conducts a safety check on the clearing energy. When the interchange schedule fails, the power-trading center should organize various market participants to modify the transaction in accordance with market rules. The proposed clearing mechanism of a two-tier electricity market is shown in Figure 2.

\section{Settlement Mechanism}

Settlement agreement may be reached on a daily basis and carried out each month in inter-provincial M-L markets. In terms of inter-provincial transactions, the NEPTC is responsible for issuing settlement basis including electricity fees, service fees, and transmission service fees. In terms of intra-provincial transactions, the PEPTC is responsible for issuing the relevant settlement basis. Provincial power grid companies carry out electricity bill settlement to market participants based on settlement basis. In terms of electricity bill payment, the purchaser pays the electricity bill to the power producer, and transmission charges are paid by both parties.

\section{MARKET-CLEARING MODEL}

The solution process of an inter-provincial market-clearing model is shown in Figure 3. The following describes the market-clearing model composition. 


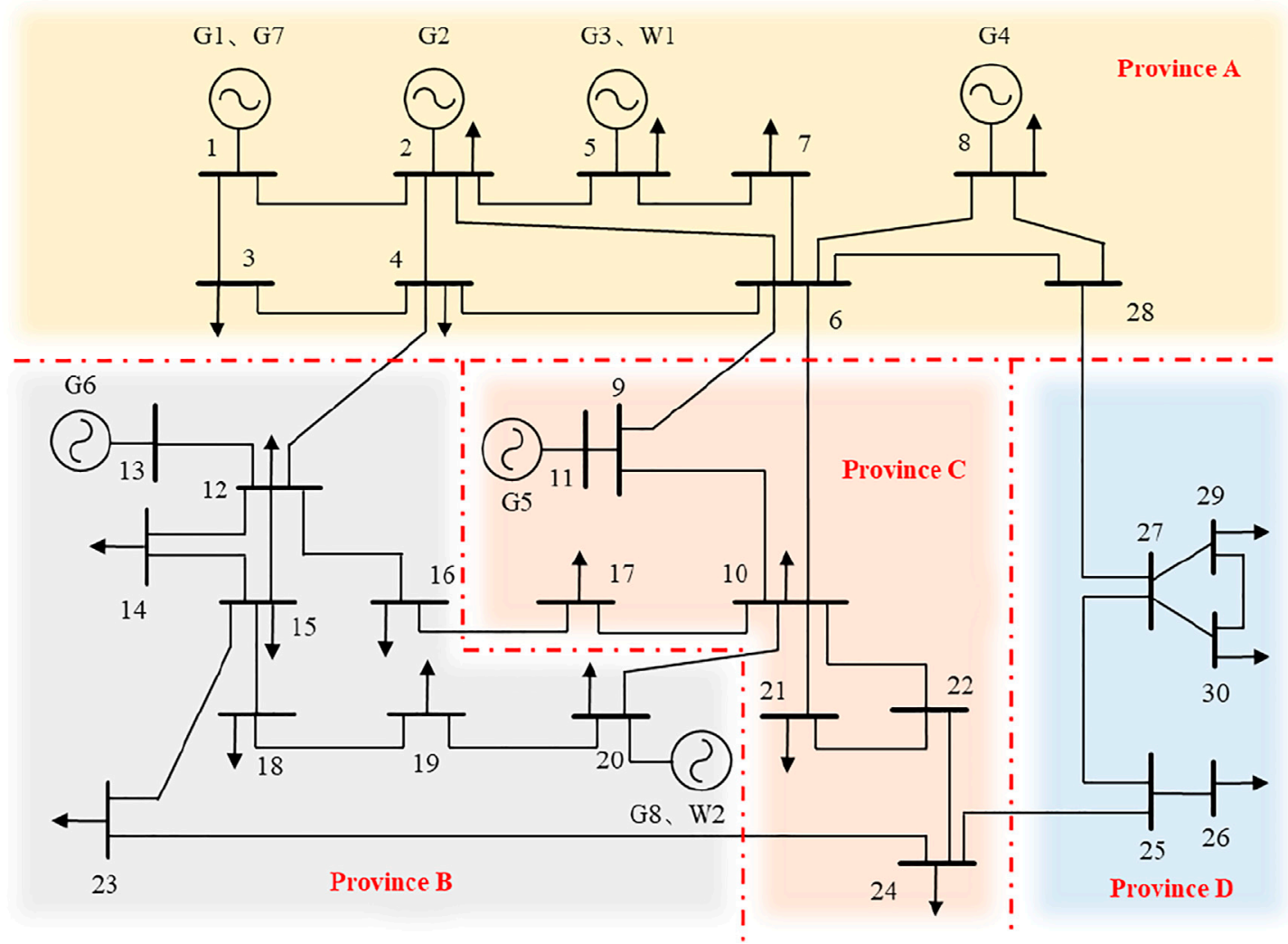

FIGURE 5 | A revised structure diagram of the IEEE 30-bus system under study.

TABLE 3 | Market-clearing energy of the proposed model considering security constraints.

\begin{tabular}{|c|c|c|c|}
\hline Clearing results & Time 1 & Time 2 & Time 3 \\
\hline Thermal unit 1/MW & 18.3 & 18.5 & 48.1 \\
\hline Thermal unit 2/MW & 12.0 & 12.0 & 12.0 \\
\hline Thermal unit 3/MW & 40.0 & 40.0 & 40.0 \\
\hline Wind unit 1/MW & 40.0 & 28.0 & 26.0 \\
\hline Wind unit 2/MW & 27.0 & 38.0 & 29.0 \\
\hline Electricity price/yuan.MWh ${ }^{-1}$ & 275.0 & 315.0 & 195.0 \\
\hline
\end{tabular}

TABLE 4 | Capacity constraints of inter-provincial electricity power transmission lines.

\begin{tabular}{lccc}
\hline Clearing results & Time 1 & Time 2 & Time 3 \\
\hline Line 1 power /MW & 22.7 & 16.0 & 25.3 \\
Line 1 ATC /MW & 17.3 & 24.0 & 14.7 \\
Line 2 power /MW & 21.3 & 22.0 & 20.7 \\
Line 2 ATC /MW & 2.7 & 2.0 & 3.3 \\
Line 3 power /MW & 1.3 & 6.0 & 4.7 \\
Line 3 ATC /MW & 14.7 & 10.0 & 11.3
\end{tabular}

\section{Power Transfer Distribution Factor}

The power transfer distribution factor (PTDF) matrix is calculated in DC power flow calculations $(\mathrm{Oh}, 2010)$. When calculating DC power
TABLE 5 | Market-clearing energy of the traditional model without considering security constraints.

\begin{tabular}{lccc} 
Clearing results & Time 1 & Time 2 & Time 3 \\
\hline Thermal unit 1/MW & 56.0 & 6.0 & 56.0 \\
Thermal unit 2/MW & 21.6 & 43.8 & 17.3 \\
Thermal unit 3/MW & 2.4 & 2.4 & 2.4 \\
Wind unit 1/MW & 12.0 & 22.4 & 20.8 \\
Wind unit 2/MW & 8.1 & 30.4 & 23.2 \\
Electricity price/yuan.MWh & & 360 & 200
\end{tabular}

flow, the linear relationship between active power injection, active power flow, and voltage angle $\theta$ can be expressed as follows:

$$
\left\{\begin{array}{l}
P_{\text {inj }}=B_{\text {bus }} \theta \\
P_{\text {flow }}=B_{\text {branch }} \theta
\end{array}\right.
$$

where $P_{\text {inj }}$ is the net injection vector, $P_{\text {flow }}$ is the power flow vector, $B_{\text {bus }}$ is the bus impedance matrix, and $B_{\text {branch }}$ is the branch impedance matrix.

When the reference bus is selected, the permutation matrix $P_{\text {ref }}$ can be constructed. Eq. 1 can be expressed as follows:

$$
P_{\text {ref }} P_{\text {inj }}=\left[\begin{array}{c}
P_{\text {inj }}^{\text {ref }} \\
P_{\text {inj }}^{\text {non-ref }}
\end{array}\right]=P_{\text {ref }} P_{\text {bus }} P_{\text {ref }}^{\mathrm{T}} P_{\text {ref }} \theta=P_{\text {ref }} P_{\text {bus }} P_{\text {ref }}^{\mathrm{T}}\left[\begin{array}{c}
\theta_{\text {inj }}^{\text {ref }} \\
\theta_{\text {inj }}^{\text {non- }}
\end{array}\right]
$$


TABLE 6 | Capacity constraints of inter-provincial lines without considering security constraints.

\begin{tabular}{lccc}
\hline Clearing results & Time 1 & Time 2 & Time 3 \\
\hline Line 1 power /MW & 37.8 & 20.0 & 44.0 \\
Line 1 ATC /MW & 12.2 & 30.0 & 6.0 \\
Line 2 power /MW & 3.4 & 13.8 & 3.3 \\
Line 2 ATC /MW & 26.6 & 16.2 & 3.3 \\
Line 3 power /MW & 41.2 & 33.8 & 47.3 \\
Line 3 ATC /MW & -21.2 & -13.8 & -27.3 \\
\hline
\end{tabular}

where ref stands for the reference bus, $P_{\text {inj }}^{\text {ref }}$ is the reference power injection, $P_{\text {inj }}{ }^{\text {non-ref }}$ is the non-reference power injection, $\theta_{\text {inj }}^{\text {ref }}$ is the reference voltage angle, and $\theta_{\text {inj }}^{\text {non-ref }}$ is the nonreference voltage.

$$
\begin{gathered}
P_{\text {inj }}^{\text {non-ref }}=\left(\begin{array}{ll}
0 & I_{N-1}
\end{array}\right) P_{\text {ref }} P_{\text {inj }}=\left(\begin{array}{ll}
0 & I_{N-1}
\end{array}\right) \\
\times\left[\begin{array}{cc}
P B P_{11}^{1 \times 1} & P B P_{12}^{1 \times(N-1)} \\
P B P_{21}^{(N-1) \times 1} & P B P_{22}^{(N-1) \times(N-1)}
\end{array}\right]\left(\begin{array}{c}
P_{\text {inj }}^{\text {ref }} \\
P_{\text {inj }}^{\text {non-ref }}
\end{array}\right) \\
=P B P_{21} \theta_{\text {inj }}^{\text {ref }}+P B P_{22} \theta_{\text {inj }}^{\text {non-ref }}
\end{gathered}
$$

where $p=P_{\text {ref }}, B=B_{\text {bus }}$, and $N$ is the number of buses.

Equation 3 can be expressed as follows:

$$
\theta_{\text {inj }}^{\text {non-ref }}=\left[P B P_{22}\right]^{-1}\left(P_{\text {inj }}^{\text {non-ref }}-P B P_{21} \theta_{\text {inj }}^{\text {ref }}\right)
$$

The second equation in Eq. 1 can be expressed as follows:

$$
\begin{aligned}
& P_{\text {flow }}=B_{\text {branch }} \theta=B_{\text {branch }} P_{\text {ref }}^{\mathrm{T}} P_{\text {ref }} \theta=B_{\text {branch }} P_{\text {ref }}^{\mathrm{T}}\left[\begin{array}{c}
\theta_{\text {inj }}^{\text {ref }} \\
\theta_{\text {inj }}^{\text {non-ref }}
\end{array}\right] \\
& =\left(\begin{array}{ll}
M^{\mathrm{ref}} & M^{\mathrm{non}-\mathrm{ref}}
\end{array}\right)\left[\begin{array}{c}
\theta_{\mathrm{inj}}^{\mathrm{ref}} \\
\theta_{\mathrm{inj}}^{\mathrm{non}-\mathrm{ref}}
\end{array}\right] \\
& =M^{\text {ref }} \theta_{\text {inj }}^{\text {ref }}+M^{\text {non-ref }} \theta_{\text {inj }}^{\text {non-ref }}
\end{aligned}
$$

where $M^{\text {ref }}$ is the reference bus term of $B_{\text {branch }} P_{\text {ref }}{ }^{\mathrm{T}}$ and $M^{\text {non-ref }}$ is the non-reference bus term of $B_{\text {branch }} P_{\text {ref }}^{\mathrm{T}}$.
According to Eqs 4, 5 can be expressed as follows.

$$
\begin{gathered}
P_{\text {flow }}=M^{\text {ref }} \theta_{\text {inj }}^{\text {ref }}+M^{\text {non-ref }}\left[P B P_{22}\right]^{-1}\left(P_{\text {inj }}^{\text {non-ref }}-P B P_{21} \theta_{\text {inj }}^{\text {ref }}\right) \\
=M^{\text {non-ref }}\left[P B P_{22}\right]^{-1} P_{\text {inj }}^{\text {non-ref }}+B P \theta_{\text {inj }}^{\text {ref }}
\end{gathered}
$$

Selecting the reference angle and setting it to zero, the linear relationship between $P_{\text {inj }}$ and $P_{\text {flow }}$ can be expressed as follows:

$$
\begin{aligned}
P_{\text {flow }}= & M^{\text {non-ref }}\left[P B P_{22}\right]^{-1} P_{\text {inj }}^{\text {non-ref }}=H^{\prime} P_{\text {inj }}^{\text {non-ref }} \\
& =\left(\begin{array}{ll}
0 & H^{\prime}
\end{array}\right)\left(\begin{array}{c}
P_{\text {inj }}^{\text {ref }} \\
P_{\text {inj }}^{\text {non-ref }}
\end{array}\right)=H P_{\text {inj }}
\end{aligned}
$$

where $H$ is the PTDF matrix and $H^{\prime}$ is the PTDF matrix without the reference bus.

The calculation method of the parameters in Eq. 7 can be expressed as follows:

$$
\begin{gathered}
\left\{\begin{array}{c}
M^{\text {non-ref }}=\operatorname{diag}(1 / X) C^{\prime} \\
P B P_{22}=C^{\prime T} \operatorname{diag}(1 / X) C^{\prime}
\end{array}\right. \\
\operatorname{diag}(1 / X)=\left[\begin{array}{cccc}
1 / x_{1} & 0 & \cdots & 0 \\
0 & 1 / x_{2} & \cdot & \cdot \\
\vdots & . & \ddots & \cdot \\
0 & . & \cdot & 1 / x_{\mathrm{L}}
\end{array}\right]
\end{gathered}
$$

where $x_{i}$ is the reactance of branch $i, C$ is the node-branch incidence, and $C^{\prime}$ is a matrix where the reference bus is eliminated. From Eqs 7-9, $H^{\prime}$ can be expressed by $C^{\prime}$ and $x$ as follows:

$$
H^{\prime}=\left[\operatorname{diag}(1 / X) C^{\prime}\right]\left\{C^{\prime T} \operatorname{diag}(1 / X) C^{\prime}\right\}^{-1}
$$

Therefore, according to Eq. 7, the PTDF matrix $H$ can be obtained.

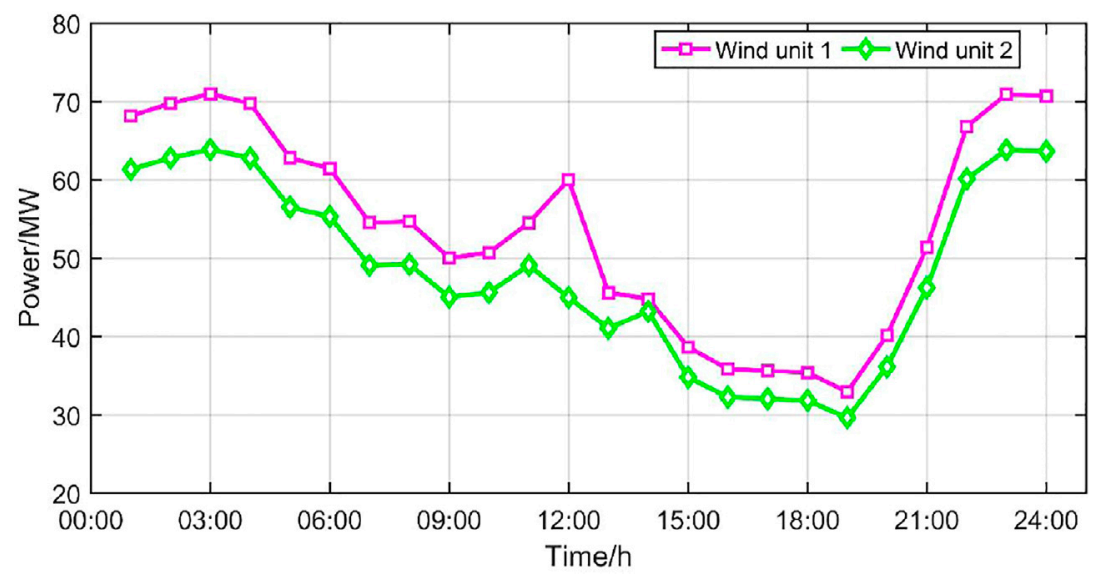

FIGURE 6 | Bidding energy of wind power producers. 


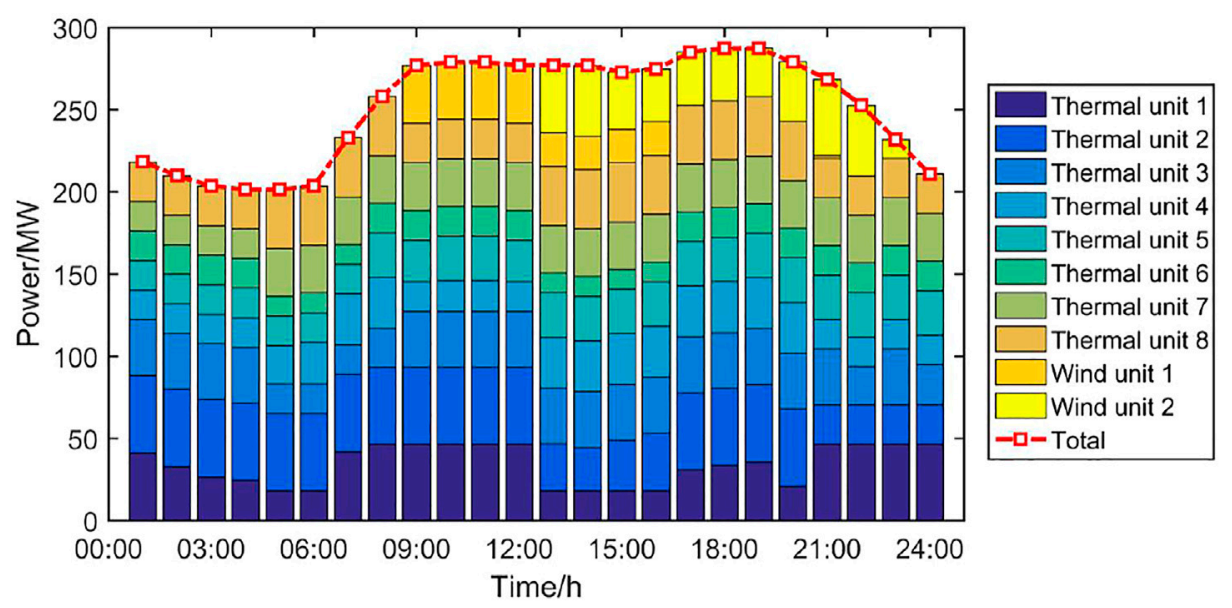

FIGURE 7 | Clearing energy of the market considering 10\% wind power capacity.

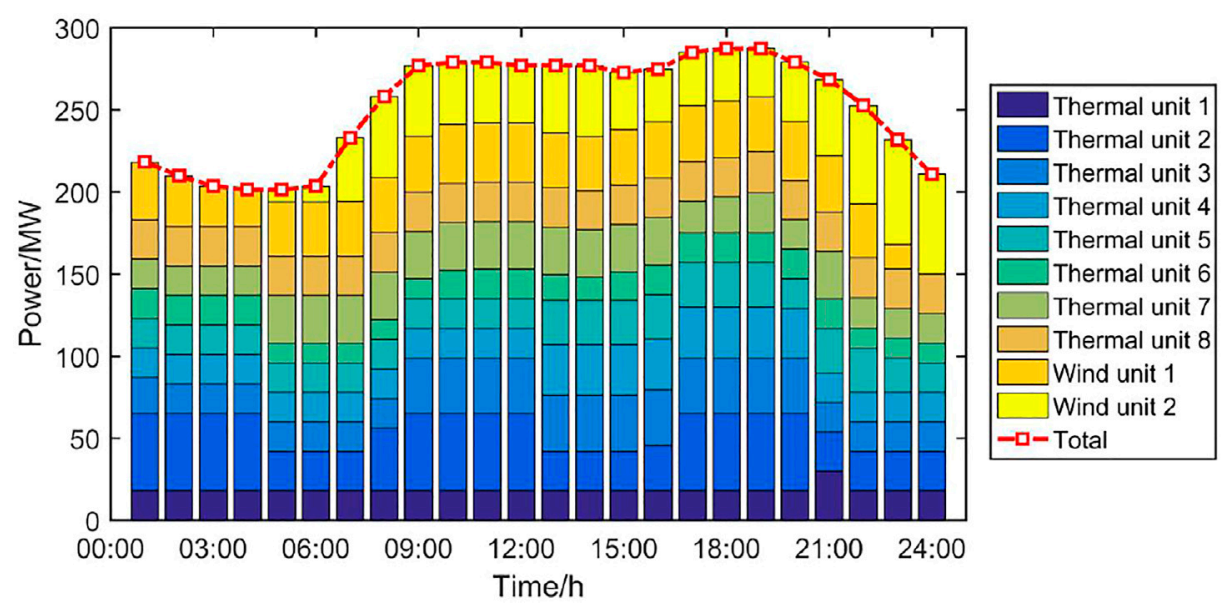

FIGURE 8 | Clearing energy of the market considering 25\% wind power capacity.

\section{Static Security Region Model}

This part establishes an inter-provincial active power static security region model based on direct DC power flow. The constraints considered by the model are generator output constraints, line DC power flow constraints, and power balance constraints (Chen et al., 2015). The model of interprovincial active power static security region $R_{S S}$ can be expressed as follows:

$$
R_{S S}=\left\{P_{G} \mid \begin{array}{c}
P_{G, \min } \leq P_{G} \leq P_{G, \text { max }} \\
-P_{L, \text { max }}+H \times P_{D} \leq H_{\mathrm{g}} \times P_{G} \leq P_{L, \text { max }}+H \times P_{D} \\
\mathrm{I}^{\mathrm{T}} P_{G}=\mathrm{I}^{\mathrm{T}} P_{D}
\end{array}\right.
$$

where $P_{G, \min }$ is the minimum generation, $P_{G, \max }$ is the maximum generation, $P_{G}$ is the scheme generation, $H$ is the power transfer distribution factor matrix, $H_{g}$ is the column in $H$ corresponding to the generator node, $P_{D}$ is the bus load vector, and $\mathrm{I}^{\mathrm{T}}$ is the unit row vector.

The safety margin of the power network can be measured by the available transfer capability (ATC) of the tie-line; ATC refers to the remaining power transmission capacity in the actual power grid that can be used for commercial use based on the existing power transmission agreement (Mohammed et al., 2019). ATC can be expressed as follows:

$$
A T C=T T C-T R M-C B M-E T C
$$

where TTC stands for total transfer capability, TRM stands for transmission reliability margin, ETC stands for existing transmission commitments, and $C B M$ stands for capacity benefit margin. 


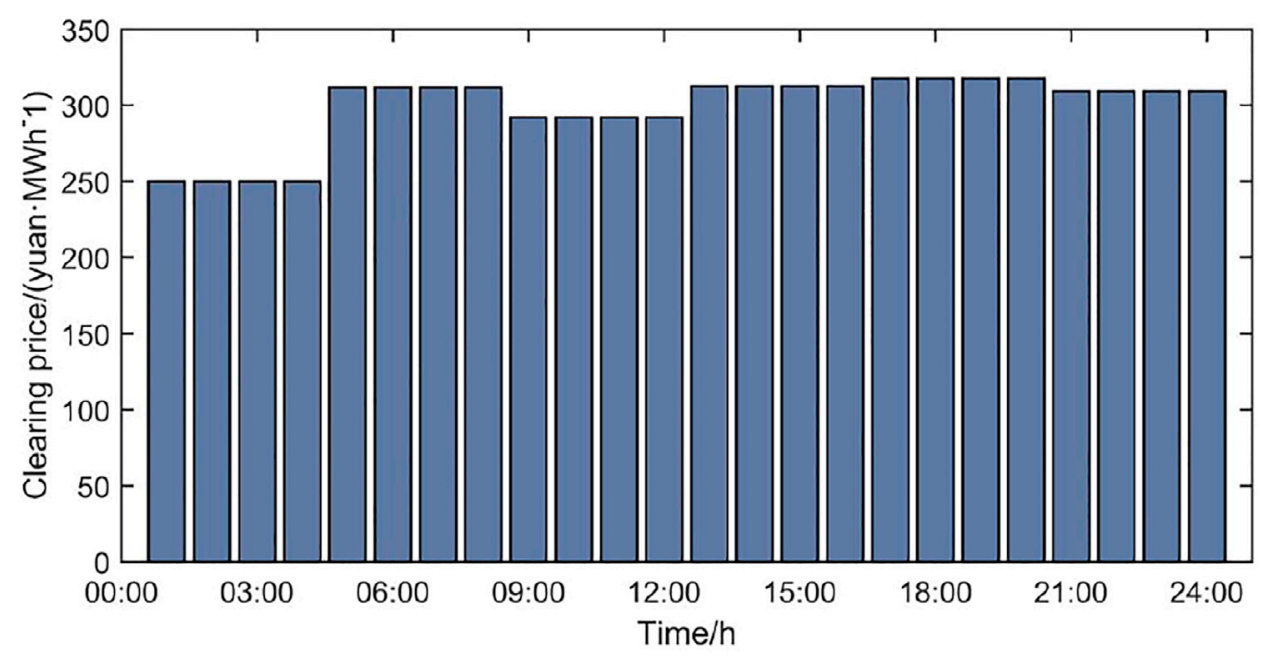

FIGURE 9 | MCP results of the simulation considering 10\% wind power capacity.

It should be noted that the power market is different from dispatching management. The concept of security region is different from the concept of security check. The foreign power market integrates market transaction and dispatching management. Safety check is a part of the marketclearing process. The electricity market operation system and dispatching management system are separately managed in China. The Market Service Organization does not have the information on system network topology, which is necessary for security check. Considering this case, we simplify the security region model. The proposed security region model is in line with the actual situation in China.

\section{Market-Clearing Model}

1) Objective function: social welfare maximization. It is expressed as follows:

$$
\begin{aligned}
\max T= & \sum_{t=1}^{\mathrm{T}} \sum_{l=1}^{\mathrm{L}} S(l, t)-\sum_{t=1}^{\mathrm{T}} \sum_{g=1}^{\mathrm{G}} S(g, t) \\
= & \sum_{t=1}^{\mathrm{T}} \sum_{l=1}^{\mathrm{L}} p_{t, l} \times c_{t, l}-\sum_{t=1}^{\mathrm{T}} \sum_{g=1}^{\mathrm{G}} p_{t, g} \times c_{t, g}+\sum_{t=1}^{\mathrm{T}} \sum_{g=1}^{\mathrm{G}} p_{t, g, u p} \times c_{t, g, u p} \\
& -\sum_{t=1}^{\mathrm{T}} \sum_{g=1}^{\mathrm{G}} p_{t, g, \text { down }} \times c_{t, g, \text { down }}-\sum_{t=1}^{\mathrm{T}} \sum_{w g=1}^{\mathrm{WG}} p_{t, w g} \times c_{t, w g}
\end{aligned}
$$

where $t$ sands for the trading time, $S(l, t)$ is the bidding energy of total consumers, $S(g, t)$ is bidding energy of the total producers, $p_{t, l}$ is the transaction electricity of the $l$ th consumer, $p_{t, g}$ is the transaction electricity of the gth generator, $p_{t, g, u p}$ is the amount of electricity increased by the gth generator, $p_{t, g \text {, down }}$ is the amount of electricity reduced by the $g$ th generator, $p_{t, w g}$ is the transaction electricity of the wind unit $w g$ at time $t, c_{t, l}$ is the bid price of the $l$ th consumer, $c_{t, g}$ is the bid price of the $g$ th generator, $c_{t, g, u p}$ is the up-spinning reserve price of the gth generator, $c_{t, g \text {, down }}$ is the down-spinning reserve price of the $g^{\text {th }}$ generator, and $c_{t, w g}$ is the bid price of the wind unit $w g$.

2) Static security constraints are given by

$$
\omega_{s s}=\left\{p \mid \begin{array}{c}
P_{g, \min } \leq p_{g} \leq P_{g, \max } \\
-P_{l, \text { min }}+H \times P_{d} \leq H_{g} \times p_{g} \leq P_{l, \text { max }}+H \times P_{d} \\
\mathrm{I}^{\mathrm{T}} p_{g}+\mathrm{I}^{\mathrm{T}} p_{w g}=\mathrm{I}^{\mathrm{T}} P_{d}
\end{array}\right.
$$

3) Wind unit output constraints are given by

$$
0 \leq p_{t, w g} \leq P_{w g, \max } \cdots \cdots \forall t \in \mathrm{T}, \forall w g \in \mathrm{WG}
$$

where $P_{w g, \text { max }}$ is the maximum output of the wind unit.

4) Generator climbing constraints are given by

$$
-S_{g, \text { up }} \leq p_{t, g}-p_{t-1, g} \leq S_{g, \text { up }} \cdots \cdots \forall t \in \mathrm{T}, \forall g \in \mathrm{G}
$$

where $S_{g \text {,up }}$ is the maximum climbing rate of generator $g$.

5) Spinning reserve constraints are given by

$$
\begin{gathered}
p_{t, g, u p}+p_{t, g} \leq P_{\mathrm{g}, \text { max }} \cdots \cdots \forall t \in T, \forall g \in \mathrm{G} \\
P_{g, \text { min }} \leq p_{t, g}-p_{t, g, \text { down }} \cdots \cdots \forall t \in T, \forall g \in \mathrm{G} \\
0 \leq p_{t, g, \text { up }} \leq K \times P_{g, \text { max }} \cdots \cdots \forall t \in T, \forall g \in \mathrm{G} \\
0 \leq p_{t, \text {, down }} \leq K \times P_{g, \text { max }} \cdots \cdots \forall t \in T, \forall g \in \mathrm{G}
\end{gathered}
$$

where $K$ is the proportional coefficient of the spinning reserve.

MCP is the average value of the bid price of the power purchase side and the power generation side in each time period. The calculation formula of the MCP in each period can be expressed as follows:

$$
P E_{t}=\frac{1}{2} \max \left(c_{t, w g}, c_{t, g}, c_{t, g, \text { down }}\right)+\frac{1}{2} \min \left(c_{t, l}, c_{t, g, u p}\right)
$$




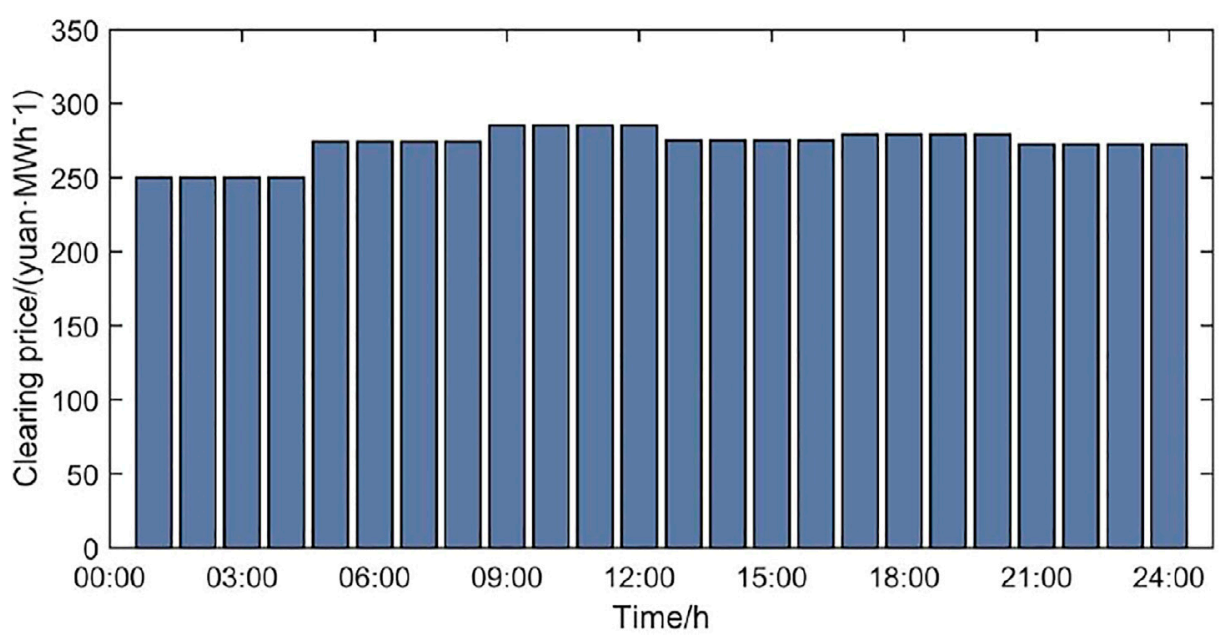

FIGURE 10 | MCP results of the simulation considering $25 \%$ wind power capacity.

\section{SIMULATION ANALYSIS}

We verify the correctness of the proposed clearing model of M-Lterm inter-provincial markets in a structure diagram of the 3-bus system and a revised structure diagram of the IEEE 30-bus system.

\section{Simulation Condition}

The structure diagram of the 3-bus power system is studied and simulated, as can be shown in Figure 4. The bidding data of the 3-bus power system can be seen in Supplementary Appendix SA. The revised structure diagram of the IEEE 30-bus system is studied and simulated, as can be shown in Figure 5. The bidding data of the IEEE 30-bus system can be found in Supplementary Appendix SB. Provinces adopt the ordinary hourly bidding method in the simulation. Customer demand and price are determined from the bidding curve on a typical day. The time granule is $1 \mathrm{~h}$. The execution, clearing, and settlement of transactions proceed using a centralized and optimized method. We combine YALMIP and CPLEX program packages to solve the market-clearing programming problems.

\section{Case 1}

In terms of the 3-bus system, each node represents a province. The nodal load represents the purchase demand. The outflow at each node represents the electricity output of a province. Lines 1 , 2 , and 3 represent inter-provincial transmission lines.

The market-clearing energy of the proposed model considering security constraints is shown in Table 3, and the maximum social welfare is 433,270 yuan. It can be seen from Table 3 that the MCPs are 275,315 , and 195 yuan/MWh, respectively. The capacity constraints of inter-provincial electricity power transmission lines are listed in Table 4, and the reliability margin of line transmission is set to $4 \%$. It can be seen from Table 4 that under the premise of the M-L transaction completion, there still exists certain amount of available transmission capacity. The remaining transmission capacity can be used for inter-provincial electricity spot trade after inter-provincial M-L trade. It can be concluded that the transaction scale can be expanded via increasing the transmission capacity of inter-provincial electricity transmission lines. Expanding interprovincial transactions can not only increase the consumption of clean energy but also obtain greater social benefits.

The following part analyzes the market-clearing results without considering security constraints. The market-clearing energy of the traditional model without considering security constraints is shown in Table 5. The capacity constraints of inter-provincial lines without considering security constraints are shown in Table 6. In this case, the maximum social welfare is 271,692 yuan. The MCPs are 310 yuan/MWh, 360 yuan/MWh, and 200 yuan/MWh. The results prove the importance of the proposed static security region model. According to the actual situation, the market clearing without considering static security region only considers the power balance constraints and unit output constraints. Table 6 lists available transmission capacities of line 3 , which has a negative value. A negative value of the transmission capacity indicates that the clearing energy exceeds the inter-provincial transmission capacity. Transmission congestion occurs when clearing energy cannot be delivered to the receiving-end power grid. The unconstrained schedule should be amended to a constrained trading schedule, which can be executed by the PEPDC and NEPDCC. This shows how static security regions are important in an inter-provincial market-clearing model. It can be concluded that the proposed market-clearing model considering system security constraints can not only ensure that transaction schedules pass the security check but also ensure fair market value in transactions.

\section{Case 2}

To further validate the proposed inter-provincial market-clearing model, the revised structure diagram of the IEEE 30-bus system is involved and simulated. The nodes with generator sets are regarded as a provincial power producer, and the remaining nodes are regarded as provincial power consumers. The bidding energy of wind power producers is shown in Figure 6.

The clearing energy of the market considering $10 \%$ wind power capacity is displayed in Figure 7, and the clearing energy of the market considering $25 \%$ wind power capacity is displayed in 
Figure 8. When the wind power penetration rate is $10 \%$, the wind power percentage of the transaction amount is $24.61 \%$. When the wind power penetration rate is $25 \%$, the wind power percentage of the transaction amount is $61.52 \%$. It can be seen from Figures 7, 8 that when the wind power penetration rate is increased, the trading of wind energy increases from 23:00 to 8:00 the next day. There are two ways for solving the problem of wind abandonment. For wind power that has not reached a deal, it can be consumed in two ways. On the one hand, energy storage devices can be built to store excess wind power. During the peak load period, the stored electrical energy can be sold to obtain additional revenue. On the other hand, local industries can be developed to consume the surplus electricity. The problem of wind abandonment is solved by two methods: interprovincial power transmission and local consumption. This will help realize the optimal allocation of renewable energy among different provinces.

The MCP results of the simulation considering $10 \%$ wind power capacity are displayed in Figure 9, and the MCP results of the simulation considering $25 \%$ wind power capacity are shown in Figure 10. When the wind power penetration rate is $10 \%$, the average MCP is 292.79 yuan/MWh. When the wind power penetration rate is $25 \%$, the average $\mathrm{MCP}$ is 272.76 yuan/MWh. Because wind power generation has low marginal cost, the MCP results of the simulation considering $10 \%$ is greater than that of the simulation considering $25 \%$. When the proportion of wind power is relatively high, there will be a negative electricity price in the power market. Therefore, while China builds power systems with a high penetration rate of renewable energy, relevant departments should establish a complete electricity price system to ensure the stable operation of the market. The MCP can reflect whether the electricity market is safe and stable. When the MCP is too high or too low, it is not in line with the real projects. The simulated MCP is compared with the actual electricity price from the Beijing Power Exchange Center to validate the performance of the proposed clearing model. According to the 2020 Market Information Disclosure Report of the Beijing Power Exchange Center, in 2020, the average MCP is 292.79 yuan/MWh, and the simulated MCP is 298.95 yuan/MWh. It can be proved that the MCP obtained by the proposed marketclearing model is consistent with the actual situation.

\section{DISCUSSION}

A system framework for building a national unified electricity market is a key to promoting the consumption of renewable energy. Optimal allocation of large-scale renewable energy can be attained via medium- and long-term transactions in different provinces. The problems of frequency regulation and peak shaving due to the uncertainty of renewable energy can be solved via flexible shortterm commercial finance solutions. The market mechanisms for promoting the use of renewable energy sources include the following areas: 1) Various types of medium- and long-term inter-provincial transaction mechanisms should be established to arrange interprovincial renewable electricity transactions. 2) The interprovincial auxiliary service mechanism should be improved to promote active participation of thermal power units to peak shaving for renewable energy. 3) An emergency support trading mechanism can be established to support power generation right trading in case of an emergency, which will probably happen due to the high proportion of renewable energy supply.

\section{CONCLUSION}

We propose a novel clearing model based on the security region for M-L inter-provincial markets. This study found that the marketclearing model considering security constraints can ensure that the constrained trading schedule passes the high-security business checks. The high penetration rate of wind power can lead to an increase in the volume of inter-provincial renewable energy trade. The research results will help improve the linkages between interprovincial markets and provide technical support for the construction of inter-provincial markets. We have the following two suggestions to help build a national unified electricity market system: 1) Both grid security and market security need to be considered in the clearing model to ensure that the clearing price meets the market security and the clearing energy meets the grid security. 2) The current electricity-trading arrangement is different in different provinces, so we need to propose a two-tier power market mechanism to break the provincial barriers.

\section{DATA AVAILABILITY STATEMENT}

The original contributions presented in the study are included in the article/Supplementary Material, further inquiries can be directed to the corresponding author.

\section{AUTHOR CONTRIBUTIONS}

XF wrote the manuscript. KY organized case studies. GL and DZ contributed to the theoretical research of this manuscript. All authors contributed to manuscript, and approved the submitted version.

\section{FUNDING}

The authors declare that this study received funding from State Grid Corporation of China (SGCC) (SGAH0000TKJS2100483). The funder was not involved in the study design, collection, analysis, interpretation of data, the writing of this article or the decision to submit it for publication.

\section{ACKNOWLEDGMENTS}

The authors would like to thank the referees and the editor of this journal for valuable comments.

\section{SUPPLEMENTARY MATERIAL}

The Supplementary Material for this article can be found online at: https:/www.frontiersin.org/articles/10.3389/fenrg.2021.839108/ full\#supplementary-material 


\section{REFERENCES}

Abhyankar, N., Lin, J., Liu, X., and Sifuentes, F. (2020). Economic and Environmental Benefits of Market-Based Power-System Reform in China: A Case Study of the Southern Grid System. Resour. Conserv. Recycl. 153, 104558. doi:10.1016/j.resconrec.2019.104558

Cai, Y., Liu, Y., Tang, X., Tan, Y., and Cao, Y. (2021). Increasing Renewable Energy Consumption Coordination with the Monthly Interprovincial Transaction Market. Front. Energ. Res. 9, 719419. doi:10.3389/fenrg. 2021.719419

Chen, S., Chen, Q., and Xia, Q. (2015). Steady-state Security Distance: Concept, Model and Meaning. Proc. Chin. Soc. Electr. Eng. 35 (03), 600-608. doi:10. 13334/j.0258-8013.pcsee.2015.03.012

Ding, Y., Xie, K., Li, Z., and Guo, L. (2020). Key Issues of National Unified Electricity Market with Chinese Characteristics (1): Enlightenment and Suggestions from Foreign Countries. Power Syst. Tech. 44 (07), 2401-2410. doi:10.13335/j.1000-3673.pst.2020.0422

Guo, L., Ding, Y., Zeng, D., and Liu, L. (2019). A Mechanism for Two-Level Power Market in China: Promoting Cross Regional Consumption of Renewable Energy. Energ. Proced. 159, 255-260. doi:10.1016/j.egypro.2018.12.059

Guo, H., Davidson, M. R., Chen, Q., Zhang, D., Jiang, N., Xia, Q., et al. (2020). Power Market Reform in China: Motivations, Progress, and Recommendations. Energy Policy 145, 111717. doi:10.1016/j.enpol.2020.111717

Jiang, T., Tan, H., Li, X., Chen, H., and Li, G. (2019). A General Optimization Model for Exploring Static Thermal Security Region Boundary in Bulk Power Systems. Proc. CSEE 39 (22), 6533-6547. doi:10.13334/j.0258-8013.pcsee.180511

Jiang, Y., Hou, J., Lin, Z., Wen, F., Li, J., He, C., et al. (2019). Optimal Bidding Strategy for a Power Producer under Monthly Pre-listing Balancing Mechanism in Actual Sequential Energy Dual-Market in China. IEEE Access 7, 70986-70998. doi:10.1109/ACCESS.2019.2919347

Jiang, T., Li, X., Li, X., Chen, H., and Li, G. (2020). General Optimization Model and Piecewise Approach for Approximating Security Region Boundary in Bulk Power Systems. Proc. CSEE 40 (14), 44114-44429. doi:10.13334/j.0258-8013. pcsee. 190884

Jin, L., Liu, W., Wang, X., Yu, J., and Zhao, P. (2021). Analyzing Information Disclosure in the Chinese Electricity Market. Front. Energ. Res. 9, 655006. doi:10.3389/fenrg.2021.655006

Li, G., Li, G., and Zhou, M. (2019). Model and Application of Renewable Energy Accommodation Capacity Calculation Considering Utilization Level of Interprovincial Tie-Line. Prot. Control. Mod. Power Syst. 4 (1), 1-12. doi:10.1186/ s41601-019-0115-7

Liu, P., Ding, T., Zou, Z., and Yang, Y. (2019). Integrated Demand Response for a Load Serving Entity in Multi-Energy Market Considering Network Constraints. Appl. Energ. 250, 512-529. doi:10.1016/j.apenergy.2019.05.003

Liu, W., Zhang, X., Wu, Y., and Feng, S. (2020). Economic Analysis of Renewable Energy in the Electricity Marketization Framework: A Case Study in Guangdong, China. Front. Energ. Res. 8, 98. doi:10.3389/fenrg.2020.00098

Pingkuo, L., Pengbo, G., and Chen, Z. (2021). How to Promote Energy Transition with Market Design: A Review on China's Electric Power Sector. Front. Energ. Res. 9, 709272. doi:10.3389/fenrg.2021.709272

Mohammed, O. O., Mustafa, M. W., Mohammed, D. S. S., and Otuoze, A. O. (2019). Available Transfer Capability Calculation Methods: A Comprehensive Review. Int. Trans. Electr. Energ. Syst. 29 (6), e2846. doi:10.1002/2050-7038.2846
Oh, H. (2010). A New Network Reduction Methodology for Power System Planning Studies. IEEE Trans. Power Syst. 25 (2), 677-684. doi:10.1109/TPWRS.2009.2036183

Song, Y., Bao, M., Ding, Y., Shao, C., and Shang, N. (2020). Review of Chinese Electricity Spot Market Key Issues and its Suggestions under the New Round of Chinese Power System Reform. Proc. CSEE 40 (10), 3172-3187. doi:10.13334/j. 0258-8013.pcsee.191251

Xia, Q., Chen, Q., Xie, K., Pang, B., Li, Z., and Guo, H. (2020). Key Issues of National Unified Electricity Market with Chinese Characteristics (2): The Development Path, Trading Varieties and Policy Recommendations of Inter-regional and Inter-provincial Electricity Markets. Power Syst. Tech. 44 (08), 2801-2808. doi:10.13335/j.1000-3673.pst.2020.0392

Xiao, J., Lin, Q., Bai, L., Zhang, X., Zuo, L., Zhou, H., et al. (2019). Security Distance for Distribution System: Definition, Calculation, and Application. Int. Trans. Electr. Energ Syst. 29 (5), e2838. doi:10.1002/2050-7038.2838

Yu, Y., Liu, Y., Qin, C., and Yang, T. (2020). Theory and Method of Power System Integrated Security Region Irrelevant to Operation States: An Introduction. Engineering 6 (7), 754-777. doi:10.1016/j.eng.2019.11.016

Zeng, M., Yang, Y., Fan, Q., Liu, Y., and Zou, Z. (2015). Coordination between Clean Energy Generation and thermal Power Generation under the Policy of "direct Power-purchase for Large Users" in China. Utilities Policy 33, 10-22. doi:10.1016/j.jup.2015.02.001

Zeng, D., Xie, K., Pang, B., Li, Z., and Yang, Z. (2020a). Key Issues of National Unified Electricity Market with Chinese Characteristics (3): Research on Transaction Clearing Models and Algorithms Adapting to the Coordinated Operation of Provincial Electricity Markets. Power Syst. Tech. 44 (08), 2809-2819. doi:10.13335/j.1000-3673.pst.2020.0435

Zeng, D., Yang, Z., Feng, S., Pang, B., and Shi, F. (2020b). Inter-provincial Power Exchange Optimization Modeling Considering ATC Constrains of Hybrid AC/ DC Power System. Power Syst. Tech. 44 (10), 3893-3899. doi:10.13335/j.10003673.pst.2020.0111

Zhang, X., and Shi, L. (2020). Future Research Areas and Key Technologies of Electricity Market in China. Automation Electric Power Syst. 44 (16), 1-11. doi:10.7500/AEPS20200602001

Zhang, K., Zhou, B., Or, S. W., Li, C., Chung, C. Y., and Voropai, N. I. (2021). Optimal Coordinated Control of Multi-Renewable-To-Hydrogen Production System for Hydrogen Fueling Stations. IEEE Trans. Ind. Applicat. 1, 1. doi:10. 1109/TIA.2021.3093841

Conflict of Interest: The authors declare that the research was conducted in the absence of any commercial or financial relationships that could be construed as a potential conflict of interest.

Publisher's Note: All claims expressed in this article are solely those of the authors and do not necessarily represent those of their affiliated organizations, or those of the publisher, the editors and the reviewers. Any product that may be evaluated in this article, or claim that may be made by its manufacturer, is not guaranteed or endorsed by the publisher.

Copyright $\odot 2022 \mathrm{Fu}$, Yang, Li and Zeng. This is an open-access article distributed under the terms of the Creative Commons Attribution License (CC BY). The use, distribution or reproduction in other forums is permitted, provided the original author(s) and the copyright owner(s) are credited and that the original publication in this journal is cited, in accordance with accepted academic practice. No use, distribution or reproduction is permitted which does not comply with these terms. 